\title{
Metabolic Disorders in Thiamineless Dwarf Strains of Staphylococcus aureus
}

\author{
By D. SOMPOLINSKY, ZIPPORA ERNST-GELLER \\ AND S. SEGAL \\ Rappaport Laboratories for Microbiology, Bar-Ilan University, Ramat-Gan, Israel
}

(Accepted for publication 28 February 1967)

\begin{abstract}
SUMMARY
Dwarf colony variants of Staphylococcus aureus have been described as a causative agent of bovine mastitis in Israel. These strains were reported as auxotrophic for thiamine, being unable to synthesize the vitamin from its pyrimidine and thiazole moieties. In the present paper it is shown that strains with two different deficiencies are involved. One type is unable to concentrate the thiazole moiety and the other is unable to phosphorylate the pyrimidine portion of the vitamin. The results indicate the existence of independent permeases in $S$. aureus for these thiamine moieties.
\end{abstract}

\section{INTRODUCTION}

Dwarf-colony variants of Staphylococcus aureus have been reported by several authors. They were generally obtained in vitro from normal strains kept under adverse conditions, such as exposure to chemicals (Hoffstadt \& Youmans, I932; Youmans \& Delves, 1942; Hale, 1947; Browning \& Adamson, 1950), to antibiotics (Barbour, 1950; Browning \& Adamson, 1950; Wise \& Spink, 1954), or to irradiation (Hale, 1949; Gause, 1961). Similar variants have been isolated from infectious lesions, especially after treatment with antibiotic drugs. In the latter case the cultures developed as normal colonies when incubated under increased $\mathrm{CO}_{2}$ pressure (Goudie \& Goudie, 1955; Thomas \& Cowlard, I955; Sherris, 1952; Hale, I95I).

We have previously reported dwarf variants of Staphylococcus aureus to cause bovine mastitis in Israel. The growth of these strains was unaffected by $\mathrm{CO}_{2}$, but when thiamine hydrochloride was added to the nutrient medium after sterilization by autoclave normal staphylococcal colonies developed (Lernau \& Sompolinsky, I962; Sompolinsky \& Levy, 196I). As known, the thiamine required for growth of $S$. aureus may normally be replaced by the pyrimidine + thiazole moieties of the vitamin (Knight, 1937). In the present communication, further studies of the metabolic disorders of these variants are reported.

\section{METHODS}

\section{Bacterial strains}

The dwarf strains were all coagulase positive and produced $\alpha$ and $\beta$ hemolysins. No differences in phage pattern and antibiotic spectra were observed between dwarf strains isolated from cows of a single herd, but strains from different herds varied.

On most nutrient media the dwarf strains formed minute transparent colonies. On 
these colonies dark orange sectors developed after a few days at $37^{\circ}$. These were composed of mutant clones which could easily be isolated and their growth was typical of Staphylococcus aureus. In some strains the dwarf colonies lying around the periphery of mutant colonies were significantly larger than when they occurred alone. This satellite growth suggested cross-feeding. This effect was not observed with other strains. Strains D 55I and D 552 were of the former type and D 1560 of the latter. These three strains were from different herds. Other dwarf strains from these herds as well as from other herds were metabolically identical with one of these two types. Only experiments with these strains will therefore be described.

Strain D I560. Phage pattern 75. For the present study we used a streptomycinresistant mutant developed in this laboratory.

Strain D 55I. Phage pattern 42 E. Some of the experiments were made with D 552 resistant to the typing phages in general use.

Strain N I560. Normally growing mutant of D I 560. The identity was checked by streptomycin resistance, phage sensitivity, and production of $\alpha$ and $\beta$ hemolysins.

For the sake of comparison several strains isolated from human pathological conditions were included in some of the experiments as described below.

\section{Media and chemicals used; cultivation}

Saline buffer: 0.I5 M- $\mathrm{Na}_{2} \mathrm{HPO}_{4}, 6 \mathrm{I} \cdot \mathrm{I} \mathrm{ml}$.; 0.15 M- $\mathrm{KH}_{2} \mathrm{PO}_{4}, 38.9 \mathrm{ml}$; $\mathrm{NaCl}, 0.8 \%$, $100 \mathrm{ml}$.

Bacto brain-heart infusion agar (BHA).

Nutrient broth prepared from: Bacto peptone, $5 \mathrm{~g}$.; beef extract (Baltimore Biol. Lab., Inc.), $3 \mathrm{~g}$.; distilled water to I 1.; $\mathrm{pH} 7 \cdot 2$.

Vitamin-free casamino acids medium (CVF): Bacto vitamin-free casamino acids, $25 \mathrm{~g}$; $\mathrm{NaCl}, 5 \mathrm{~g}$.; nicotinic acid, I mg.; distilled water to I 1.; $\mathrm{pH} 7 \cdot 2$.

Defined medium, modified from Gladstone (1937): $\mathrm{KH}_{2} \mathrm{PO}_{4}, 0.9 \mathrm{I}$ g.; $\mathrm{Na}_{2} \mathrm{H} \mathrm{PO}_{4}$, 3.83 g.; $\mathrm{Mg} \mathrm{SO}_{4} .7 \mathrm{H}_{2} \mathrm{O}$, 0.13 g.; $\mathrm{Fe} \mathrm{SO}_{4}$. $\left(\mathrm{NH}_{4}\right)_{2} \mathrm{SO}_{4} .6 \mathrm{H}_{2} \mathrm{O}$, 0.01 g.; glucose, $5.0 \mathrm{~g}$; nicotinic acid, $\mathrm{I} \cdot 0 \mathrm{mg}$; ; distilled water to $\mathrm{I} 1 . ; \mathrm{pH} 7 \cdot 4$. The following amino acids were added: glycine, L-valine, L-leucine, L-proline, L-aspartic acid, L-glutamic acid to a final concentration of I/I500 M; L-phenylalanine, L-arginine, L-histidine to I/4000 M; $\mathrm{L}$-cystine to $\mathrm{I} / \mathrm{I} 0000 \mathrm{M}$. Thiamine or its thiazole and pyrimidine moieties were added as indicated. The $\mathrm{KH}_{2} \mathrm{PO}_{4}, \mathrm{Na}_{2} \mathrm{HPO}_{4}$ and $\mathrm{MgSO}_{4} \cdot 7 \mathrm{H}_{2} \mathrm{O}$ were autoclaved, the other ingredients were sterilized by filtration and added aseptically.

Thiamine hydrochloride (Sigma), thiamine pyrophosphate (Sigma), sodium pyruvate, A grade (Calbiochem), 2-methyl 4-amino 5-aminomethylpyrimidine from $\mathrm{E}$. Merck, Darmstadt, were used and 4-methyl-5-( $\beta$ hydroxyethyl)-thiazole synthesized by Professor Dr B. Prijs, Institute for Inorganic Chemistry, Basel University, Switzerland. The two thiamine moieties will be referred to as the pyrimidine and the thiazole moieties.

Phosphorylated derivatives of the thiazole and pyrimidine moieties. Due to lack of authentic samples the following preparations were used. Staphylococcal strain N I 560 was grown under aeration in CVF medium without thiamine, and the starved cocci harvested, washed and transferred to Warburg respiration vessels (see later). To one vessel was added $6 \times 10^{-3} \mathrm{M}$ of the pyrimidine moiety, to another an equimolar amount of the thiazole moiety. The vessels were incubated at $37^{\circ}$ for $20 \mathrm{hr}$. After separation of the cocci, the supernatant solutions were designated 'crude pyrimidine pyrophos- 
phate' and 'crude thiazole phosphate', respectively. It was assumed that these solutions probably contained a certain amount of phosphorylated end-products synthesized by the cocci. The solutions were virtually free of thiamine, since they did not stimulate oxygen consumption with pyruvate by starved bacteria of the strain N I560.

Growth. To determine growth requirements, the cultures were grown with aeration in a water bath at $37^{\circ}$ for $20 \mathrm{hr}$. in CVF medium enriched with $3 \times 10^{-9} \mathrm{M}$-thiamine. The cocci were harvested, washed five times in cold $0.05 \mathrm{M}$ phosphate-buffer $\left(\mathrm{pH}_{7} \cdot 0\right)$, resuspended in Gladstone's defined medium to a concentration giving 20 Klett units (filter $540 \mathrm{~m} \mu$ ) and incubated for $24 \mathrm{hr}$. Growth was indicated by an increase in turbidity of at least 100 Klett units.

Oxygen uptake was measured by standard Warburg techniques, $Q_{\mathrm{o}_{2}}$ values are expressed as $\mu \mathrm{l} . \mathrm{O}_{2} / \mathrm{hr} / \mathrm{mg}$. bacterial dry wt. The cocci were grown in broth, harvested, washed five times in saline buffer $\left(\mathrm{pH}^{\circ}{ }^{\circ}\right)$ and resuspended to a concentration equivalent to 600-900 Klett units. Bacterial dry weight was determined on samples dried for $18 \mathrm{hr}$ at $105^{\circ}$. Each Warburg vessel contained $0.5 \mathrm{ml}$. of phosphate buffer as above and I ml. of bacterial suspension. The centre well contained $0.2 \mathrm{ml} .20 \% \mathrm{KOH}$. After $20 \mathrm{~min}$. incubation, $0.5 \mathrm{ml}$. of a sodium pyruvate solution was added from the side arm. With a $0.1 \mathrm{M}$ solution ( $50 \mu$ moles) respiratory activity was maximal and linear during the first $30-50 \mathrm{~min}$., with $\mathrm{I} \cdot \mathrm{M}$ the linear period extended for several hours (Fig. I). Oxygen uptake reached values of $15-22 \mu$ moles $\mathrm{O}_{2} / 50 \mu$ moles pyruvate, which approached the theoretical value in oxidative decarboxylation. The endogenous respiration was $8-12 \%$ of that found with an exogenous substrate.

\section{RESULTS}

\section{Growth requirements}

Growth of staphylococcal strain N I560 depended on supplementation of Gladstone's defined medium with either thiamine or with both moieties of the vitamin. When the medium was enriched with thiamine both types of dwarf variants grew as well. Strain D 55I gave no growth on defined medium supplemented with the thiazole or the pyrimidine singly or together even at high concentrations. On the other hand, strain D I 560 grew as well as strain N 1560 when both the thiazole and pyrimidine were added together, provided that the thiazole concentration was about I0,000 times higher than that required by strain N I560 (Tables I, 2). Further experiments showed that the pyrimidine requirement for growth was quantitatively similar in strains N I560 and D I560.

\section{Respiration with pyruvate as substrate}

Further information on the enzymic defects which cause the thiamine requirement of the dwarf variants was obtained by measuring the respiration of oxygen uptake with pyruvate as substrate, in Warburg manometers under various conditions.

Strain N I560. When grown in nutrient broth, the cocci of the normal mutant showed a marked $\mathrm{O}_{2}$ uptake with pyruvate as substrate. Addition of thiamine had no influence on respiration. The same was true for six recently isolated human strains of Staphylococcus aureus. This indicates that the cocci after growth in nutrient broth had an intracellular store of thiamine or thiamine equivalent (e.g. cocarboxylase).

On the other hand, the cocci of strain N I 560 were depleted of thiamine, and they 


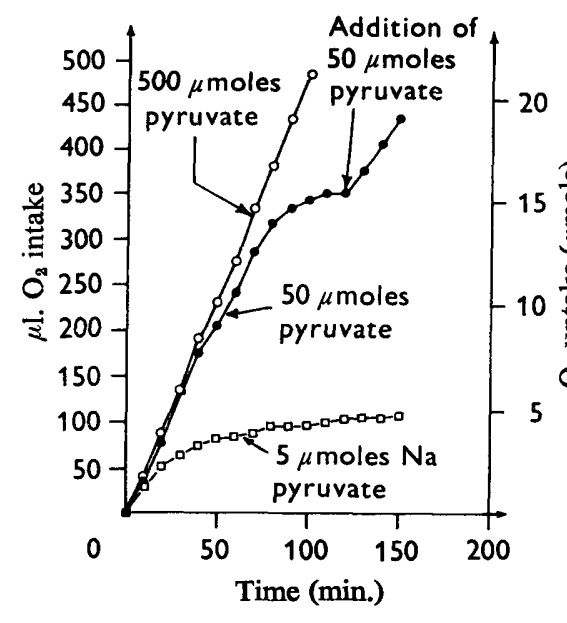

Fig. I

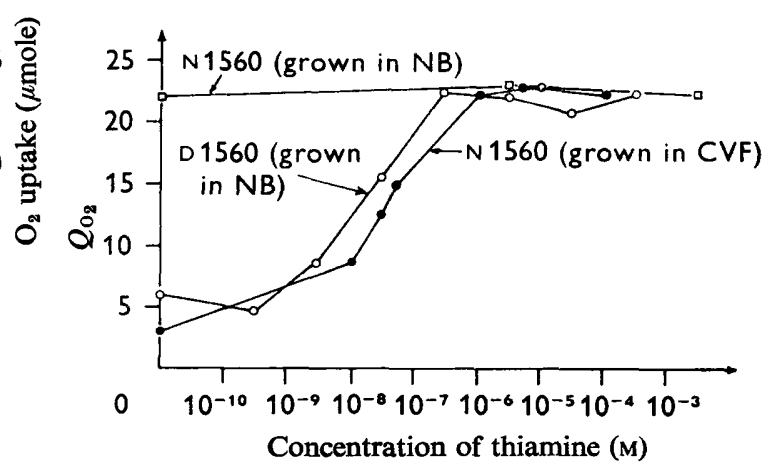

Fig. 2

Fig. I. Dependence of oxygen uptake by Staphylococcus aureus D I 560 on the amount of sodium pyruvate added. Each vessel contained $2.5 \mu \mathrm{g}$. thiamine hydrochloride. $\square-\square, 5 \mu$ mole pyruvate; - , $50 \mu$ mole pyruvate, a further $50 \mu$ mole added at 120 min.; $\mathrm{O}-\mathrm{O}, 500$ $\mu$ mole pyruvate.

Fig. 2. Relationship between oxygen uptake $\left(Q_{O_{2}}\right)$ with pyruvate as substrate by Staphylococcus aureus and the thiamine concentration. $\square-\square$, strain $N$ I 560 grown in nutrient broth (NB); - - , strain N1560 grown in vitamin free casamino acid medium; $O-O$, strain D 1560 grown in nutrient broth (NB).

Table I. Growth of dwarf variants and a normal (mutant) strain of Staphylococcus aureus in Gladstone's (1937) defined medium with addition of thiamine or its moieties

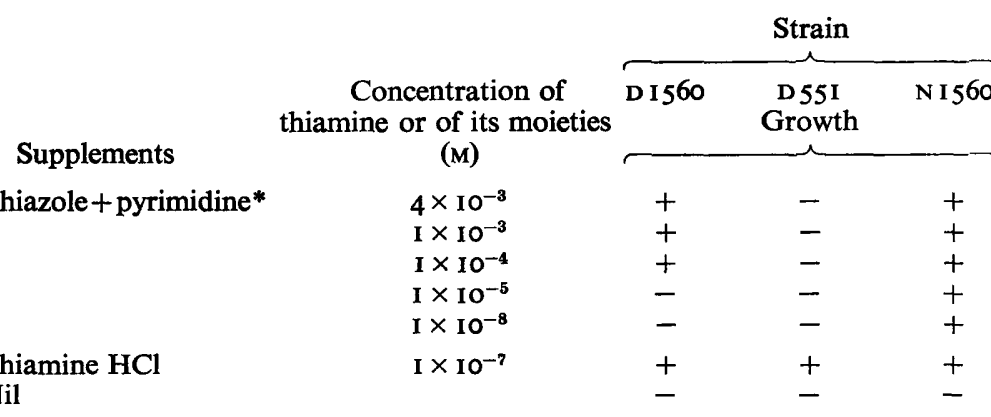

* The thiazole and pyrimidine moieties of thiamine.

Table 2. Growth of a dwarf variant (D I560) of Staphylococcus aureus in Gladstone's defined medium supplemented with the thiamine moieties

$\begin{array}{lccc}\text { Thiazole }(\mathrm{M})^{*} & \text { Pyrimidine }(\mathrm{M})^{*} & \text { Thiamine }(\mathrm{M}) & \text { Growth } \\ \mathrm{I} \times 1 \mathrm{I}^{-3} & \mathrm{I} \times 1 \mathrm{O}^{-5} & . & + \\ \mathrm{I} \times 1 \mathrm{O}^{-5} & \mathrm{I} \times \mathrm{IO}^{-8} & \cdot & - \\ \mathrm{I} \times 1 \mathrm{O}^{-3} & \cdot & \mathrm{I} \times 1 \mathrm{O}^{-7} & - \\ \cdot & \cdot & . & + \\ \text { No supplement } & \cdot & \end{array}$

* The thiazole and pyrimidine moieties of thiamine. 
respired with pyruvate as substrate only when thiamine was added to the Warburg vessel, when the bacteria had been grown in CVF medium without supplementation or when supplemented with only a minute amount of thiamine $\left(3 \times 10^{-10} \mathrm{M}\right)$. The relation between $Q_{\mathrm{o}_{2}}$ and thiamine concentration was linear in the range of approximately $10^{-8}-10^{-7} \mathrm{M}$ (Fig. 2). Respiration was likewise stimulated by addition to the Warburg vessel of both moieties of thiamine together at similar concentrations. When growth in CVF medium was maximal, the cocci were starved for both these compounds.

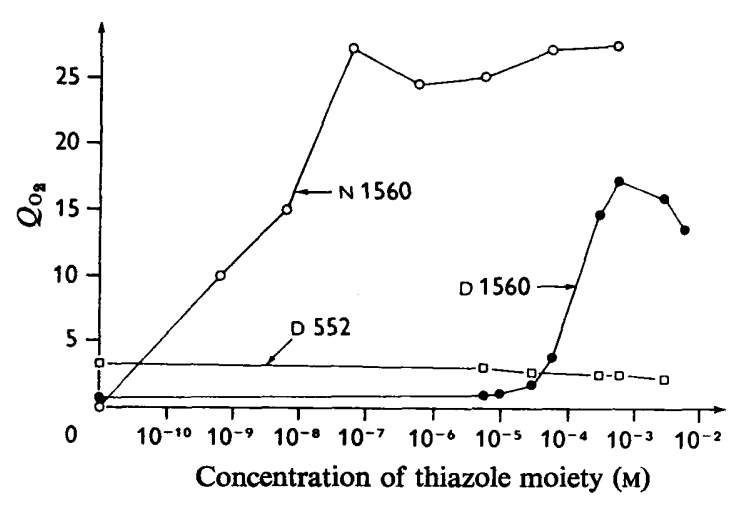

Fig. 3

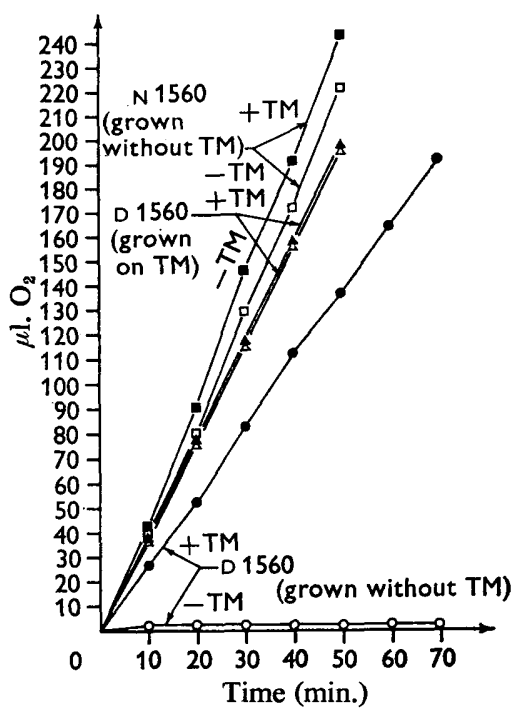

Fig. 4

Fig. 3. Relation between oxygen uptake with pyruvate as substrate by different Staphylococcus aureus strains and concentration of the thiazole moiety of thiamine. Concentration of the pyrimidine moiety was $6 \times 10^{-4} \mathrm{M}$ throughout. Strain N1560 was deficient in thiamine after growth in vitamin free casamino acid medium. $\square-\square$, strain D 552; -0 , strain D I 560; O-O, strain N I560.

Fig. 4. Oxygen uptake with pyruvate as substrate by Staphylococcus aureus, strain N 1560 and strain DI560 with and without addition of extraneous thiamine (TM) $\left(3 \times 10^{-6} \mathrm{M}\right)$. $\square-$, Strain N 1560 grown without thiamine, with thiamine added. $\square-\square$, strain N I560 grown without thiamine, without thiamine. $\Delta-\Delta$, strain D I560 grown with thiamine, with thiamine added; $\triangle-\triangle$, strain DI560 grown with thiamine, without thiamine. strain D 1560 grown without thiamine, with thiamine added; $O-O$, strain D I560 grown without thiamine, without thiamine.

By variation of the concentration of the pyrimidine with a constant concentration of the thiazole and vice versa, it was found that the requirement for both moieties was approximately equivalent to the required concentration of thiamine (Fig. 3). Respiration was maximal and linear from the beginning of the experiment, indicating a high ability of the non-proliferating cocci to concentrate the pyrimidine and the thiazole and to synthesize cocarboxylase.

Strain D 1560. This variant, when grown in nutrient broth, was unable to respire with pyruvate as substrate unless thiamine was added. The concentration of thiamine needed was equivalent to that required by strain N 1560 after growth in CVF medium. Supplementation of nutrient broth with thiamine had the effect that the harvested 
cocci respired with pyruvate as substrate, without addition of more thiamine (Fig. 4).

Stimulation of oxygen uptake was obtained to the same extent when the thiazole moiety was substituted for thiamine, but the concentration required was approximately 500 times higher, on a molar basis. The pyrimidine had no influence on respiration with strain D I 560, not even at concentrations required by strain N I560 (Fig. 5). The 'crude thiazole phosphate' preparation did not stimulate respiration when added at concentrations significantly lower than those at which the thiazole moiety was effective, at higher concentrations its effect on oxygen consumption was as with the unphosphorylated thiazole compound.

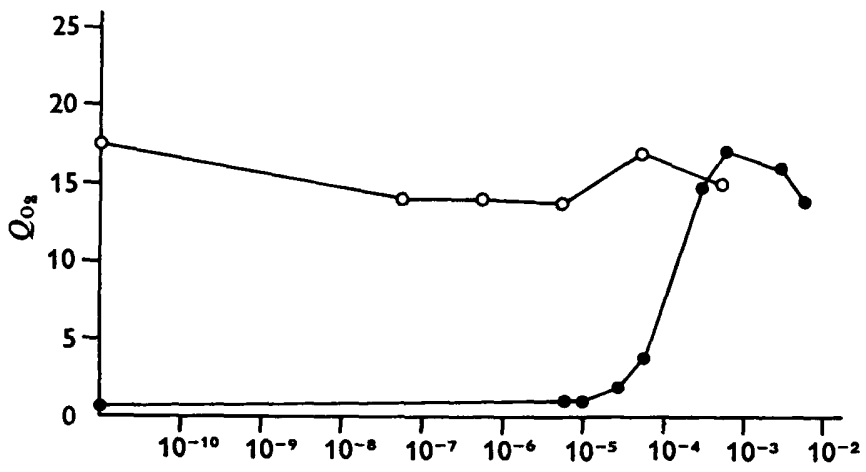

Concentration of pyrimidine and thiazole moieties (M)

Fig. 5. Oxygen uptake with pyruvate as substrate by Staphylococcus aureus D 1560 , grown in nutrient broth. $\mathrm{O}-\mathrm{O}$, Concentration of the thiazole moiety of thiamine $6 \times 10^{-4} \mathrm{M}$; concentration of the pyrimidine moiety is shown on the abscissa. -1 , Concentration of pyrimidinemoiety $6 \times 10^{-4} \mathrm{M}$; concentration of thiazole moiety shown on the abscissa.

Table 3. Stimulation of respiration of strain D $55 \mathrm{I}$ by 'crude thiazole phosphate' and 'crude pyrimidine pyrophosphate'

Supplement (M) added to Warburg vessel

$3 \times 10^{-6}$ thiamine

None

$4 \times 10^{-5}$ crude thiazole phosphate $+6 \times 10^{-4}$ pyrimidine moiety

$4 \times 10^{-6}$ crude thiazole phosphate

$4 \times 10^{-6}$ crude thiazole phosphate $+6 \times 10^{-4}$ pyrimidine moiety

$2 \times 10^{-5}$ crude pyrimidine pyrophosphate

$2 \times 10^{-5}$ crude pyrimidine pyrophosphate $+6 \times 10^{-4}$ thiazole moiety

$2 \times 10^{-6}$ crude pyrimidine pyrophosphate

$2 \times 10^{-6}$ crude pyrimidine pyrophosphate $+6 \times \mathrm{IO}^{-4}$ pyrimidine moiety

$2 \times 10^{-8}$ crude pyrimidine pyrophosphate

$3 \times 10^{-8}$ thiamine $+6 \times 10^{-3}$ pyrimidine moiety

* Relative $Q_{\mathrm{O}_{2}}$ values are calculated as $\%$ of the $Q_{\mathrm{O}_{2}}$ observed with $3 \times 10^{-6} \mathrm{M}^{*}$-thiamine.

Strains $D 55 I$ and $D 552$. These variants behaved exactly like strain D I 560 with respect to requirement for thiamine after growth in nutrient broth. On the other hand, neither the pyrimidine moiety nor the thiazole moiety, nor both together, stimulated oxygen uptake at any concentration up to $4 \times 10^{-3} \mathrm{M}$. However, a significant stimulation was obtained with the 'crude pyrimidine pyrophosphate' preparation at a concen- 
tration corresponding to $2 \times 10^{-6} \mathrm{M}$ pyrimidine. Oxygen uptake with the 'crude pyrimidine phosphate' was never more than $25-50 \%$ of that obtained with thiamine and was strongly antagonized by the unphosphorylated pyrimidine. The thiazole moiety or the 'crude thiazole phosphate' did not stimulate $\mathrm{O}_{2}$ uptake by themselves and had no influence on $\mathrm{O}_{2}$ uptake when added to the 'crude pyrimidine phosphate' (Table 3).

\section{DISCUSSION}

Cocarboxylase synthesis by Staphylococcus aureus (Tsubota, 1964) follows the same pathway as that in baker's yeast (Camiener \& Brown, 1960). This may be outlined as follows:

$\left.\begin{array}{l}\text { Pyrimidine moiety } \rightarrow \text { pyrimidine pyrophosphate } \\ \text { Thiazole moiety } \rightarrow \text { thiazole phosphate }\end{array}\right\} \rightarrow\left\{\begin{array}{c}\text { thiamine phosphate } \rightarrow \\ \text { thiamine } \rightarrow \text { thiamine } \\ \text { pyrophosphate }\end{array}\right.$

Since stimulation of respiration by starved cocci of strain N I560 with exogenous thiazole and pyrimidine moieties of thiamine was maximal from the moment the substrate (pyruvate) was added, the synthesis of cocarboxylase is apparently rapid, and was probably performed during the pre-incubation period ( $20 \mathrm{~min}$, see Methods).

During growth in unsupplemented nutrient broth the normal strain, N 1560, builds up an intracellular reserve of thiamine or thiamine pyrophosphate. This reserve must be synthesized from the thiazole and pyrimidine moieties, since thiamine present in the nutrient medium is destroyed during sterilization. The strain D I 560 was deficient for thiamine after growth in nutrient broth, growth of this strain depended probably on traces of thiamine or on a slow synthesis of the vitamin from its breakdown products. The cocci needed extraneous thiazole at a molar concentration about 500 times that of thiamine for maximal respiratory activity. It is, however, of interest that this strain concentrated the pyrimidine moiety intracellularly during growth in nutrient broth, and needed no extraneous supply of this compound for respiration of pyruvate (Fig. 5).

The failure to concentrate thiazole could be explained by $(a)$ a defect in the enzyme which phosphorylates the thiazole moiety, or $(b)$ lack of permease for this compound. Proof of the first assumption would automatically deny the existence of a permease for the thiazole moiety, since otherwise a defective phosphorylating enzyme would lead to a maximal packing of the cocci with the thiazole moiety. But the fact that respiration of strain D55I with 'crude pyrimidine phosphate' was independent of extraneous thiazole suggests that in this strain (and probably also in other Staphylococcus aureus strains) a permease system for the thiazole moiety does exist. The second explanation is therefore the more likely, and is also in accordance with the fact that 'crude thiazole phosphate' did not stimulate respiration of strain D I 560 at concentrations significantly lower than that needed when the nonphosphorylated thiazole was used.

Strain D 55I was probably deficient in enzyme which converts the pyrimidine moiety to the pyrimidine pyrophosphate. This is indicated by $(a)$ failure to decarboxylate pyruvate when given thiazole + pyrimidine moieties even at high concentration, and (b) stimulation of oxygen uptake by the product designated 'crude pyrimidine pyrophosphate', which logically should contain a certain amount of the phosphorylated pyrimidine. 
The antagonistic effect of non-phosphorylated pyrimidine on 'crude pyrimidine pyrophosphate' might most probably indicate competition for a permease, since the non-phosphorylated compound should have no influence on the thiamine phosphate synthesizing enzyme. That this antagonism was not merely due to a contamination of our pyrimidine compound with some inhibitory chemical was demonstrated by its failure to inhibit the stimulation by thiamine of pyruvate respiration by the same strain (D 55I). The suggestion that a permease for the pyrimidine moiety does exist in Staphylococcus aureus is also consistent with the intracellular gathering of this compound by the cells of strain D I560 during growth in nutrient broth (Fig. 5). Although these results offer no conclusive proof, they seem to indicate that permeases for the pyrimidine moiety and the thiazole moiety are present in Staphylococcus aureus. These two permease systems are most probably independent of each other since cocci of strain D I560, which are unable to concentrate the thiazole moiety, nevertheless have enough intracellular pyrimidine moiety for co-enzyme synthesis. The existence of a permease for thiamine in Lactobacillus fermenti has recently been shown in a convincing way (Neujahr, I966).

The authors are indebted to Professor B. Prijs (Basel University) for the generous gift of 4-methyl-5-( $\beta$-hydroxyethyl)-thiazole and to Dr S. Yurugi, Research and Development Division, Takeda Chemical Industries, Ltd., Osaka, Japan, and to Dr J. S. Tzoumertis, Merck Sharp and Dohme Research Laboratories, Rahway, New Jersey, U.S.A., who kindly provided other samples of thiazoles and pyrimidines. Warm appreciation is expressed to Dr H. Lernau, V.M.D., of the Laboratory for Milk Hygiene, Veterinary Institute, Bet Dagon, Israel, for giving us the opportunity to examine D variants isolated by him, as well as for his constant interest in the work, and to Professor A. Pinsky for his help and critical advice in preparing the manuscript.

A part of this investigation was supported by Research Grant 66/8I to Z. E. G. from Bar-Ilan University.

\section{REFERENCES}

BARBOUR, R. G. H. (1950). Small colony variants ('G' forms) produced by Staphylococcus pyogenes during the development of resistance to streptomycin. Aust. J. exp. Biol. med. Sci. 28, 41 I.

BrownING, D. H. \& Adamson, H. S. (1950). Stable dwarf colony forms produced by Staphylococcus pyogenes. J. Path. Bact. 62, 499.

CAmiener, G. W. \& Brown, G. M. (1960). The biosynthesis of thiamine. II. Fractionation of enzyme system and identification of thiazole monophosphate and thiamine monophosphate as intermediates. J. biol. Chem. 235, $241 \mathrm{I}$.

GAUSE, G. F. (I96I). Biochemical changes accompanying impaired respiration in Staphylococcus aureus. Nature, Lond. r90, 978.

Gladstone, G. P. (1937). The nutrition of Staphylococcus aureus: nitrogen requirements. Br. J. exp. Path. 18, 322.

Goudie, J. G. \& Goudie, R. B. (1955). Recurrent infections by a stable dwarf-colony variant of Staphylococcus aureus. J. clin. Path. 8, 284.

HALE, J. H. (1947). Studies on staphylococcus mutation: characteristics of the G (gonidial) variant and factors concerned in its production. Br. J. exp. Path. 28, 202.

HALE, J. H. (1949). Studies on staphylococcus mutation: an investigation of the growth requirements of a 'G' (gonidial) variant. Br. J. exp. Path. 30, 297.

Hale, J. H. (I95I). Studies on staphylococcus mutation: a naturally occurring ' $G$ ' (gonidial) variant and its carbon dioxide requirements. Br. J. exp. Path. 32, 307. 
Hoffstadt, R. E. \& Youmans, G. P. (1932). Staphylococcus aureus dissociation and its relation to infection and immunity. J. inf. Dis. 51, 216.

KNIGHT, B. C. J. G. (1937). The nutrition of Staphylococcus aureus. The activities of nicotinamide, aneurin (vitamin B I), and related compounds. Biochem. J. 31, 966.

LERNAU, H. \& SOMPOLINSKY, D. (1962). Bovine mastitis due to dwarf colony variants of Staphylococcus aureus. Cornell Vet. 52, 445.

Neujatr, H. Y. (1966). Transport of B-vitamins in micro-organisms. III. Chromatographic studies on the radioactivity extracted from non-proliferating cells of Lactobacillus fermenti after exposure to labelled thiamine. Acta chem. scand. 20, 786.

ShERris, J. C. (1952). Two small colony variants of Staphylococcus aureus isolated in pure culture from closed infected lesions and their carbon dioxide requirements. J. clin. Path. 5, 354 .

Sompolinsky, D. \& LeVy, M. (I96I). Further studies on the metabolism of D-colony forms of Staphylococcus aureus. Bull. Res. Coun. Israel, sect. E, 9, 3.

Thomas, M. E. M. \& Cowlard, J. H. (1955). Studies on a $\mathrm{CO}_{2}$-dependent Staphylococcus. J. clin. Path. 8, 288.

Tsubota, Y. (1964). Studies on the enzymatic synthesis of thiamine by S. aureus. Bitamin (Kyoto) 29, 510 (Japan). Quoted from Chem. Abstr., 1965, 62, 1990.

Wise, R. J. \& SpINK, W. W. (1954). The influence of antibiotics on the origin of small colonies (G variants) of Micrococcus pyogenes var. aureus. J. clin. Invest. 33, 1611 .

Youmans, G. P. \& Delves, E. (1942). The effect of inorganic salts on the production of small colony variants by Staphylococcus aureus. J. Bact. 44, 127. 